\title{
All methods on deck
}

\author{
More basic research studies of marine microorganisms - supported by new methods, tools and resources - are \\ needed to help inform policies to mitigate the impact of climate change.
}

A s humans, we have long been drawn to the ocean. We lounge on the beach, surf the waves and sail on the wind. We voraciously consume its delicacies, from the humble oyster to the swift bluefin tuna. In our living rooms we are glued to nature documentaries illuminating the hugeness of blue whales, the intelligence of dolphins, the ferocity of great white sharks and the strange, colorful beauty of corals.

This abundance of the ocean rests on the very smallest of organisms - microbial life forms, from viruses to multicellular zooplankton. These microorganisms do not capture the public's interest the way that so many ocean creatures do. But they are fundamental to the health of the ocean, and the planet: they represent the ocean's predominant biomass, the bottom of the marine food web, and, in the case of phytoplankton, the major producers of atmospheric oxygen. They are also the 'first responders' to environmental change. Last June, a large group of microbiologists published a Consensus Statement in Nature Reviews Microbiology declaring that the "microscopic majority can no longer be the unseen elephant in the room" and highlighting the central role of marine microorganisms in regulating the increasing threat of climate change.

There is still just so much that is unknown to science about ocean life in general - and so much opportunity. For example, a recent Comment in Nature urged researchers to focus on the ecosystem of the understudied twilight zone, that perpetually dim region found at a depth of 200-1,000 meters under the surface. And this month in Nature Methods, we are pleased to publish a Resource describing tools for genetically manipulating strategically chosen marine protists, selected to represent key taxa on the eukaryotic tree. These tools will enable a deeper understanding of the cell biology, biogeochemical roles and evolutionary history of these diverse and

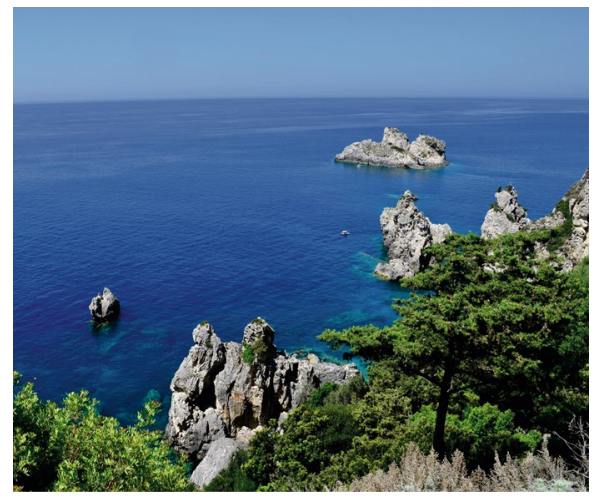

Credit: Allison Doerr

difficult-to-study unicellular eukaryotes.

(Also read the News and Views.)

The vastness, diversity, changing conditions and inaccessibility of the ocean generates real methodological challenges for studying its life forms. For example, a researcher studying the human gut microbiome faces enough hardship worrying about a relatively small, contained environment - whereas a marine microbiologist must contend with cubic miles of dynamically changing sample space. Temperatures, currents, $\mathrm{pH}$, salinity and disruptions like storms, ships and pollution cause ocean ecosystems to undergo daily and seasonal variation, requiring long-term collection of time-series data. There are countless different environments and different scales at which organisms operate in such environments, and the majority of organisms are impossible to study in the controlled setting of a lab.

Ocean scientists use a wide array of tools and methods, ranging from the collection of water samples from cruises, to robots that trawl the ocean floor, to fixed and floating sampling stations, to satellites used for remote sensing. (Journalist Vivien Marx describes some of these tools in a recent Technology Feature.) These approaches generate huge amounts of data in terms of bytes, but in the grand view of the ocean, the datasets are extremely sparse. International collaboration is needed to cover broader areas, and data sharing is essential. Computational integration of datasets, collected using different techniques and at different scales, represents another challenge. Ocean studies often require an interdisciplinary approach, combining expertise in biology, physics, chemistry, geology, engineering and computational modeling - disciplines that need more communication with each other.

A greater fundamental scientific understanding of the ocean and its most basic biological processes will help inform polices to protect habitats and to mitigate the effects of climate change, as well as to support human populations that depend on the ocean. A joined-up, global effort may help facilitate this: the United Nations has named the 2020s as the Decade of Ocean Science for Sustainable Development. The goal of this ambitious project is to connect stakeholders from around the globe via a common framework to "reverse the cycle of decline of ocean health" and create "improved conditions for sustainable development of the ocean."

Nature Methods is committed to publishing a diverse selection of methods for basic biology research, but most of the papers we publish have a decidedly biomedical bent - a new sequencing approach could be applied in human disease studies, for example, or a new cryo-EM method could be used in a quest to design drugs. Yet climate change is one of the most pressing issues facing humanity, and thus we hope to support research in this area by publishing and highlighting more papers describing methods, tools and resources for studying life - especially the microscopic majority - in the ocean.

Published online: 5 May 2020

https://doi.org/10.1038/s41592-020-0846-4 